PHYSICAL REVIEW D 95, 069906(E) (2017)

\title{
Erratum: Spectroscopic analysis of stellar mass black-hole mergers in our local universe with ground-based gravitational wave detectors [Phys. Rev. D 94, 084024 (2016)]
}

\author{
Swetha Bhagwat, Duncan A. Brown, and Stefan W. Ballmer \\ (Received 26 February 2017; published 29 March 2017)
}

DOI: 10.1103/PhysRevD.95.069906

In Ref. [1], we claim that the prospects for measuring multiple modes of the ringdown of a binary black-hole merger are promising with the future ground-based detectors but that it is unlikely with the Advanced LIGO, even at design sensitivity. Our analysis used analytical fits to the black-hole ringdown mode amplitudes presented in Ref. [2]. The formula of Ref. [2] contained an error, and our manuscript used corrected fits given in private communication with London [3]. Subsequently, London et al. have published an erratum [4] with the corrected fits. We checked our implementation of the mode amplitude fitting formula against the published erratum and have discovered an error in our code compared to the formula in the erratum. The amplitudes of the modes $A_{21}$ and $A_{33}$ given in Eqs. (3) and (4) of Ref. [3] are a factor of 2 greater than the values that we used in our analysis. As a result, our analysis was too conservative, and the prospect of measuring multiple ringdown modes increases significantly. When we repeat the analysis described in Ref. [1] with the corrected amplitudes, we find that Advanced LIGO will detect about three events per year that are spectroscopically valuable, assuming an optimistic rate of $240 \mathrm{Gpc}^{-3} \mathrm{yr}^{-1}$ merger events. Fixing the error in our mode amplitudes also changes our quantitative

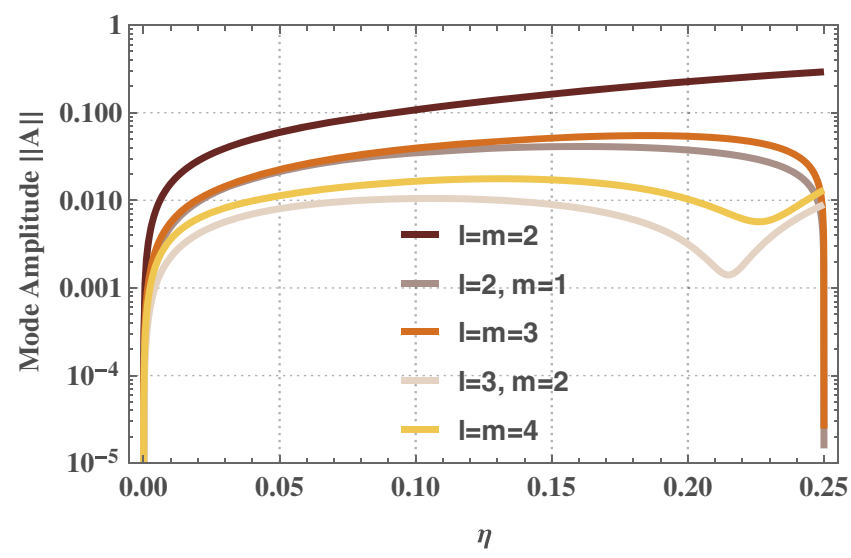

FIG. 1. This figure presents the magnitude of mode amplitudes $\|A\|$ predicted by the fitting formulas given in Ref. [2] as a function of dimensionless symmetric-mass-ratio $\eta$. Comparing the amplitudes of different modes, we infer that the potential candidates for subdominant mode measurability correspond to $l=m=3, l=2, m=1$, and $l=m=4$.

TABLE I. The results we obtain from a Mont Carlo simulation of $10^{6}$ stellar mass binary black-hole mergers uniformly distributed in component mass, orientation, and volume defined by a sphere of radius $1500 \mathrm{Mpc}$. We categorize each event into one of the sets defined below and tabulate the fraction of signals that falls into each set. Set 1: the $l=m=2$ mode could not be detected. Set 2: the $l=m=2$ could be detected, but no other subdominant mode could be detected. Set 3: the $l=m=3$ subdominant mode can be measured. Set 4: the $l=m=4$ subdominant mode can be measured. Set 5: both $l=m=3$ and $l=m=4$ subdominant modes can be measured. Set 6 : there was failed measurability of subdominant mode due to the resolvability criterion.

\begin{tabular}{llllccc}
\hline \hline Detector curve & Set 1 & Set 2 & Set 3 & Set 4 & Set 5 & Set 6 \\
\hline Advanced LIGO & 0.83 & 0.16 & 0.0008 & $2 \times 10^{-5}$ & $1.6 \times 10^{-5}$ & 0 \\
A+ & 0.6 & 0.37 & 0.01 & 0.0004 & 0.0001 & 0 \\
Einstein telescope & 0.32 & 0.47 & 0.18 & 0.03 & 0.01 & $2 \times 10^{-4}$ \\
Cosmic explorer & 0.3 & 0.44 & 0.3 & 0.08 & 0.04 & $5 \times 10^{-6}$ \\
A flat noise curve at $10^{-25} \sqrt[2]{\mathrm{Hz}}^{-1}$ & 0.17 & 0.35 & 0.41 & 0.06 & 0.18 \\
\hline \hline
\end{tabular}


results. We present updated Tables 1-4 in this erratum. Figure 1 in this erratum presents a corrected version of Fig. 2 of Ref. [1].

The qualitative results that compare the $l=m=3$ with the $l=2, m=1$ mode in the published version are correct because this error does not affect the amplitude ratio of $A_{21}$ and $A_{33}$.

TABLE II. Information similar to Table I but with sets defined differently. Set 1: the $l=m=2$ mode could not be detected. Set 2: the $l=m=2$ could be detected, but the $l=2, m=1$ subdominant mode could be detected. Set 3: the $l=2, m=1$ subdominant mode was both detected and resolved. Set 4: the $l=2, m=1$ subdominant mode was detected but not resolved. Here, again, we tabulate the number of events out of $10^{6}$ Monte Carlo simulated binary black-hole mergers that fall in each of these sets.

\begin{tabular}{|c|c|c|c|c|}
\hline Detector curve & Set 1 & Set 2 & Set 3 & Set 4 \\
\hline Advanced LIGO & 0.84 & 0.15 & $1.8 \times 10^{-4}$ & 0 \\
\hline $\mathrm{A}+$ & 0.59 & 0.4 & 0.004 & 0 \\
\hline Einstein telescope & 0.31 & 0.52 & 0.16 & 0 \\
\hline Cosmic explorer & 0.24 & 0.41 & 0.34 & 0 \\
\hline A flat noise curve at $10^{-25} \sqrt[2]{\mathrm{Hz}}^{-1}$ & 0.14 & 0.33 & 0.52 & 0 \\
\hline
\end{tabular}

TABLE III. Using our results in Table I and the optimistic (pessimistic) rates of binary black-hole mergers, predicted based on the recent discoveries of binary black-hole mergers [5], at $240 \mathrm{Gpc}^{-3} \mathrm{yr}^{-1}\left(13 \mathrm{Gpc}^{-3} \mathrm{yr}^{-1}\right)$, we present the rate of events that would allow measurability of the $l=m=3$ or $l=m=4$ subdominant mode with current and future ground-based detectors. We present this combined $(l=m=3$ or $l=m=4)$ rate because detuning the detector around the frequency band 300-500 Hz for a ringdown-oriented search benefits both of these modes.

\begin{tabular}{lcc}
\hline \hline Detector curve & Optimistic rate & Pessimistic rate \\
\hline Advanced LIGO & $3 / \mathrm{yr}$ & $0.2 / \mathrm{yr}$ \\
A+ & $47 / \mathrm{yr}$ & $3 / \mathrm{yr}$ \\
Einstein telescope & $694 / \mathrm{yr}$ & $38 / \mathrm{yr}$ \\
Cosmic explorer & $1130 / \mathrm{yr}$ & $61 / \mathrm{yr}$ \\
A flat noise curve at $10^{-25} \sqrt[2]{\mathrm{Hz}}-1$ & $1570 / \mathrm{yr}$ & $85 / \mathrm{yr}$ \\
\hline \hline
\end{tabular}

TABLE IV. Using our results in Table II and the optimistic (pessimistic) rate of binary black-hole mergers, predicted based on the recent discoveries of binary black-hole mergers [5], at $240 \mathrm{Gpc}^{-3} \mathrm{yr}^{-1}\left(13 \mathrm{Gpc}^{-3} \mathrm{yr}^{-1}\right)$, we present the rate of events that would allow measurability of single subdominant modes.

\begin{tabular}{|c|c|c|}
\hline For $l=m=3$ & Optimistic rate & Pessimistic rate \\
\hline Advanced LIGO & $3 / y r$ & $0.2 / \mathrm{yr}$ \\
\hline $\mathrm{A}+$ & $46 / y r$ & $3 / \mathrm{yr}$ \\
\hline Einstein telescope & $645 / \mathrm{yr}$ & $35 / y r$ \\
\hline Cosmic explorer & $1024 / \mathrm{yr}$ & $56 / y r$ \\
\hline A flat noise curve at $10^{-25} \sqrt[2]{\mathrm{Hz}^{-1}}$ & $1409.2 / \mathrm{yr}$ & $76.3 / \mathrm{yr}$ \\
\hline For $l=m=4$ & Optimistic rate & Pessimistic rate \\
\hline Advanced LIGO & $0.08 / \mathrm{yr}$ & $0.004 / \mathrm{yr}$ \\
\hline $\mathrm{A}+$ & $1.4 / \mathrm{yr}$ & $0.08 / \mathrm{yr}$ \\
\hline Einstein telescope & $97 / \mathrm{yr}$ & $5 / \mathrm{yr}$ \\
\hline Cosmic explorer & $263 / y r$ & $14 / y r$ \\
\hline A flat noise curve at $10^{-25} \sqrt[2]{\mathrm{Hz}^{-1}}$ & $605.7 / \mathrm{yr}$ & $32.8 / \mathrm{yr}$ \\
\hline For $l=2, m=1$ & Optimistic rate & Pessimistic rate \\
\hline Advanced LIGO & $0.6 / \mathrm{yr}$ & $0.03 / \mathrm{yr}$ \\
\hline $\mathrm{A}+$ & $13 / \mathrm{yr}$ & $0.7 / \mathrm{yr}$ \\
\hline Einstein telescope & $546 / \mathrm{yr}$ & $30 / y r$ \\
\hline Cosmic explorer & $1163 / \mathrm{yr}$ & $63 / y r$ \\
\hline A flat noise curve at $10^{-25} \sqrt[2]{\mathrm{Hz}^{-1}}$ & $1772.3 / \mathrm{yr}$ & $96 / y r$ \\
\hline
\end{tabular}


[1] S. Bhagwat, D. A. Brown, and S. W. Ballmer, Phys. Rev. D 94, 084024 (2016).

[2] L. London, D. Shoemaker, and J. Healy, Phys. Rev. D 90, 124032 (2014).

[3] L. T. London (private communication).

[4] L. T. London, J. Healy, and D. Shoemaker, Phys. Rev. D 94, 069902 (2016).

[5] B. P. Abbott et al. (LIGO Scientific Collaboration and Virgo Collaboration), www.dcc.ligo.org/P1600088, 2016. 